\title{
Usability and Patient Preference Phase 3 Study of the Sarilumab Pen in Patients with Active Moderate- to-Severe Rheumatoid Arthritis
}

\author{
Alan Kivitz · Lydie Baret-Cormel · Hubert van Hoogstraten · Sheldon Wang • \\ Janie Parrino $\cdot$ Christine $\mathrm{Xu} \cdot$ Marina Stanislav
}

Received: October 25, 2017 / Published online: December 5, 2017

(C) The Author(s) 2017. This article is an open access publication

\begin{abstract}
Introduction: Sarilumab is a human monoclonal antibody that blocks the interleukin-6 receptor alpha (IL-6R $\alpha$ ). The phase 3 SARIL-RAEASY study (EASY) assessed the robustness of an autoinjector (pen) for administering sarilumab when used by adults with active moderate-tosevere rheumatoid arthritis (RA) who are candidates for anti-IL-6R therapy in an unsupervised real-world setting.

Methods: EASY was a 12-week, multicenter, randomized, open-label, parallel-group usability study of the sarilumab pen and prefilled syringe.
\end{abstract}

Enhanced content To view enhanced content for this article go to www.medengine.com/Redeem/ A7FCF060054F5676.

A. Kivitz ( $\square)$

Altoona Center for Clinical Research, Duncansville, PA, USA

e-mail: ajkivitz@yahoo.com

L. Baret-Cormel

Sanofi, Paris, France

H. van Hoogstraten · S. Wang · C. Xu

Sanofi Genzyme, Bridgewater, NJ, USA

J. Parrino

Regeneron Pharmaceuticals, Inc, Tarrytown, NY, USA

M. Stanislav

V.A. Nassonova Research Rheumatology Institute, Moscow, Russia
Patients were randomized 1:1:1:1 to sarilumab 150 or $200 \mathrm{mg}$ every 2 weeks (q2w) administered via pen or syringe, plus background disease-modifying antirheumatic drugs. Patients reported their ability to remove the pen cap and initiate and complete injections; negative responses were defined as product technical complaints (PTCs). The primary endpoint was the number of validated product technical failures (PTFs; PTC with a validated technical cause). This study was not powered to demonstrate bioequivalence or differences in efficacy among groups.

Results: A total of 217 patients were randomized. There were 600 successful injections with the sarilumab pen in 108 patients and no penassociated PTFs. One PTC was observed (the pen was mistakenly activated before injection). At week $12,88 \%$ of patients indicated the pen was "easy" to use, and 98\% reported they were "satisfied" with the pen. Proportions of patients achieving an American College of Rheumatology 20/50/70 response and a 28-joint disease activity score by C-reactive protein $<2.6$ were similar at each dose between the pen and syringe groups, as were the pharmacokinetics. There were no clinically meaningful differences in adverse events (AEs), serious AEs, and AEs leading to discontinuation in the pen and syringe groups. The most common treatmentemergent AEs were infections and neutropenia. Conclusion: This study demonstrated the ease of use and robustness of the sarilumab pen 
when used by patients with RA in an unsupervised setting. Pharmacokinetics, safety, and efficacy were generally similar for the pen and syringe groups (NCT02057250).

Funding: Sanofi Genzyme and Regeneron Pharmaceuticals, Inc.

Trial Registration: Clinicaltrials.gov identifier, NCT02057250.

Keywords: Autoinjector; IL-6; IL-6R $\alpha$; Patient preference; Pen; Prefilled syringe; Rheumatoid arthritis; Sarilumab

\section{INTRODUCTION}

Rheumatoid arthritis (RA) is a chronic, progressive, autoimmune disease characterized by joint inflammation and pain, morning stiffness, and progressive joint destruction [1]. Blockade of interleukin 6 (IL-6) signaling is a therapeutic option for the treatment of RA [2-9]. Sarilumab is a human IgG1 monoclonal antibody that binds specifically to both soluble and membrane-bound IL-6 receptors (sIL-6R $\alpha$ and mIL$6 \mathrm{R} \alpha$ ), and has been shown to inhibit IL-6-mediated signaling through these receptors [2-4]. Sarilumab has been approved for the treatment of moderate-to-severe RA in the United States, Canada, and Europe [10-12].

As lifelong treatment of RA is required to reduce joint damage progression and conserve health-related quality of life, physical function, and work productivity, more convenient forms of therapy could facilitate compliance. Patients with RA may encounter challenges associated with the use of syringes due to the functional limitations associated with the disease [13]. To address patient grip and dexterity limitations, a single-use, disposable, prefilled, buttonless, ergonomic autoinjector (pen) for the
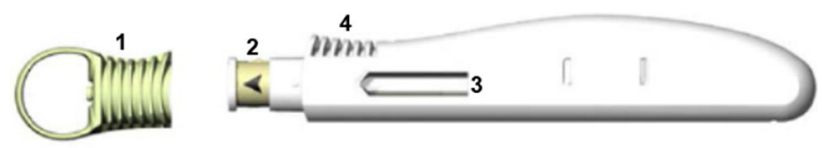

Fig. 1 Sarilumab pen. Visual features of the prefilled, single-use, disposable, buttonless, ergonomic sarilumab pen include (1) an easy-to-remove needle cap, (2) a needle cover that is automatically positioned after injection to administration of sarilumab was designed. The SARIL-RA-EASY study (EASY; NCT02057250) primarily assessed the robustness of this prefilled pen for administering sarilumab when used by adults with active moderate-to-severe RA in an unsupervised real-world setting. Secondary objectives were to assess patient satisfaction when using the pen and to compare the pharmacokinetics (PK) of sarilumab administered by pen and by syringe.

\section{METHODS}

\section{Pen Design}

The single-use, disposable, ergonomic sarilumab pen has an easy-to-remove needle cap, a needle cover that is automatically positioned after injection to help prevent needlestick injuries, a window that enables the patient to see the medication and provides visual feedback during the injection (the transparent window turns yellow when the injection is complete), strips to facilitate grip, and audible clicks that signal the beginning and end of the injection (Fig. 1). The pen has buttonless activation to facilitate the injection (i.e., depression of the spring-loaded plunger against the skin triggers the expulsion of the drug). The sarilumab pen only serves as a delivery device and contains the same sarilumab prefilled syringe as that used for comparison in this study.

\section{Study Design}

EASY was a phase 3, multicenter, global, randomized, open-label, 12-week study in patients with active moderate-to-severe RA to assess the robustness and usability of the sarilumab pen (Fig. 2). After completion, patients were allowed help prevent needlestick injuries, (3) a transparent window that turns yellow when the injection is complete, and (4) strips to facilitate grip 


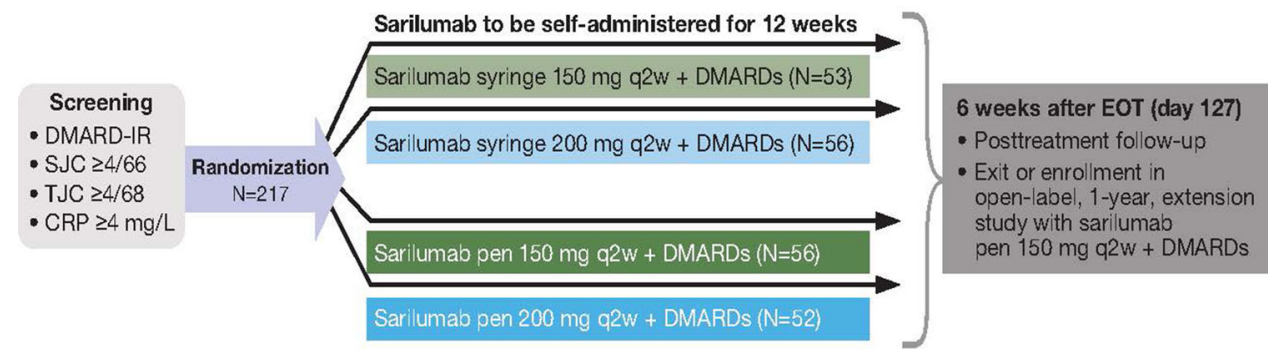

Fig. 2 EASY study design. Patients completing the 12-week study (assessment phase) could elect to participate in a 1-year open-label extension phase. CRP C-reactive

to enroll in an open-label, 1-year extension study. Patients were randomized 1:1:1:1 to sarilumab 150 or $200 \mathrm{mg}$ every 2 weeks (q2w) administered via pen or syringe, plus background disease-modifying antirheumatic drugs (DMARDs). All patients were to self-administer all doses via subcutaneous (SC) injection in either the abdomen or thigh. In exceptional circumstances when the patient was unable to self-inject, a caregiver could administer the injection in the upper arm. This study was not powered to determine bioequivalence, as no meaningful differences were expected between the devices.

The protocol was approved by the appropriate ethics committees/institutional review boards (ECs/IRBs), and each patient gave written informed consent. The study was conducted in compliance with IRB regulations, the International Conference on Harmonization Guidelines for Good Clinical Practice, and the Declaration of Helsinki. The study (MSC12665) is registered with Clinicaltrials.gov (NCT02057250).

\section{Patient Population}

Eligible patients were $\geq 18$ years of age at baseline and fulfilled the 2010 American College of Rheumatology/European League Against Rheumatism (ACR/EULAR) Rheumatoid Arthritis Classification Criteria with a disease duration of $\geq 12$ weeks [14] and a functional status of ACR class I-III, based on the 1991 revised criteria [15]. Patients were included if they had active moderate-to-severe RA defined as $\geq 4$ of protein, DMARD disease-modifying antirheumatic drug, $E O T$ end of treatment, $I R$ inadequate response, $q 2 w$ every 2 weeks, SJC swollen joint count, TJC tender joint count

66 swollen joints and $\geq 4$ of 68 tender joints, and high-sensitivity C-reactive protein (hs-CRP) $\geq 4 \mathrm{mg} / \mathrm{L}$. Patients had to be willing and able to self-inject for the duration of the study. In addition, patients had to have been continuously treated with one or a combination of nonbiologic DMARDs (excluding the simultaneous use of leflunomide [LEF] and methotrexate [MTX]) for $\geq 12$ consecutive weeks before randomization and to have been on a stable dose for $\geq 6$ consecutive weeks before screening of any of the following: MTX (10-25 mg/week), LEF (10-20 mg orally daily), sulfasalazine (1000-3000 mg orally daily), or hydroxychloroquine (200-400 mg orally daily). Patients treated within 12 weeks of baseline with any DMARD other than those allowed per protocol, which were limited to the maximum specified dosage, were excluded.

\section{Assessments}

The subset of patients assigned to use the sarilumab pen recorded evaluations of their experience using the pen after each injection in home diaries, including answering questions as to whether they were able to remove the pen cap and initiate and complete injections. A response of "no" to any of these three questions was defined as a product technical complaint (PTC). Product technical complaints identified from patient diaries were collected and sent with the corresponding pen to the sponsor for evaluation and technical analysis. The primary endpoint was the number of validated product technical failures (PTFs), defined as a PTC with a 
validated technical cause. Diaries were reviewed at weeks $4,6,8,10$, and 12 to assess any problems associated with the pen during administration. Patients who were assigned to use the syringe were not required to maintain diaries.

Patient perception and satisfaction were assessed via a two-part questionnaire. The first part was completed at baseline to assess perceptions regarding injections and prior experience with self-injection. The second part was completed at week 12 to assess experiences performing self-injection with the sarilumab pen during the study.

The concentration-time profiles of sarilumab in serum with the pen and syringe were compared after the first dose (weeks 0-2) and at the sixth dose (weeks 10-12, representing a steady state). Pharmacokinetic parameters were determined using noncompartmental analysis of serum concentration data. Other endpoints included safety (adverse events [AEs] and clinical laboratory evaluations) and efficacy parameters (proportions of patients achieving an ACR20/50/70 response and a 28-joint disease activity score by CRP [DAS28-CRP] $<2.6$ at week 12).

\section{Statistical Analysis}

All randomized patients who received at least one dose of the study drug and attended at least one postbaseline visit were included in the modified intent-to-treat population, while all randomized patients who received at least one dose of the study drug were included in the safety population. The sample size for this study was based on practical considerations and clinical judgment rather than formal sample size calculations. Data were analyzed using descriptive statistics.

\section{RESULTS}

\section{Patient Disposition and Characteristics}

Of the 217 patients initially randomized to receive sarilumab 150 or $200 \mathrm{mg} \mathrm{q} 2 \mathrm{w}$ plus DMARDs via pen or syringe, 201 (92.6\%) completed the assessment phase. Completion rates were similar among the groups: $92.9 \%$ and $94.3 \%$ for the pen and syringe $150 \mathrm{mg}$ groups, respectively, and $86.5 \%$ and $96.4 \%$ for the pen and syringe $200 \mathrm{mg}$ groups, respectively. Sixteen $(7.4 \%)$ patients discontinued from the study. The most common reason for discontinuation (5.5\% of patients overall) was AEs in each of the treatment groups. No clinically meaningful differences were observed between AEs in the pen and syringe groups or between dose groups. Baseline demographic and disease characteristics were similar among groups (Table 1).

\section{Sarilumab Pen-Related Endpoints}

There were a total of 600 successful injections with the sarilumab pen in 108 patients, for an overall rate of 5.6 injections per patient. The number of successful injections with the pen was similar in the two dose groups (312 injections of $150 \mathrm{mg}$ in 56 patients and 288 injections of $200 \mathrm{mg}$ in 52 patients). There were no pen-associated PTFs. One PTC occurred. The PTC was considered a user-related error, as the patient accidentally bumped the pen after pulling the cap off, which triggered the injection and expelled the drug onto the floor.

\section{Patient Perception and Satisfaction}

At baseline, before the first injection, the majority of patients reported that they were not afraid of needles (58\%), had past experience with self-injections (55\%), and were either "very confident" or "extremely confident" regarding self-injections (55\%). After the 12-week assessment phase, when asked about their overall level of satisfaction, $98 \%$ of patients reported they were "satisfied" or "very satisfied" with the sarilumab pen. Most patients (88\%) indicated the sarilumab pen was "easy" or "very easy" to use; 98\% thought the injection time was either "normal," "short," or "very short;" and 91\% were "very confident" to "extremely confident" about using the same pen for self-injection in the future (Table 2). 
Table 1 Baseline demographic and disease characteristics

\begin{tabular}{|c|c|c|c|c|}
\hline & \multicolumn{4}{|c|}{ Sarilumab q2w + DMARDs } \\
\hline & $\begin{array}{l}\text { Pen } 150 \mathrm{mg} \\
(N=56)\end{array}$ & $\begin{array}{l}\text { Syringe } 150 \mathrm{mg} \\
(N=53)\end{array}$ & $\begin{array}{l}\text { Pen } 200 \mathrm{mg} \\
(N=52)\end{array}$ & $\begin{array}{l}\text { Syringe } 200 \mathrm{mg} \\
(N=56)\end{array}$ \\
\hline Age, mean (SD), years & $53.7(13.8)$ & $54.2(14.2)$ & $55.9(12.3)$ & $50.3(12.8)$ \\
\hline Female, $n(\%)$ & $45(80.4)$ & $43(81.1)$ & $44(84.6)$ & $49(87.5)$ \\
\hline Weight, mean (SD), kg & $78.3(17.5)$ & $79.1(19.1)$ & $78.1(17.1)$ & $77.1(24.6)$ \\
\hline White, $n(\%)$ & $54(96.4)$ & $46(86.8)$ & $47(90.4)$ & $45(80.4)$ \\
\hline $\begin{array}{l}\text { Prior biologic DMARD use for } \\
\text { RA, } n(\%)\end{array}$ & $14(25.0)$ & $13(24.5)$ & $17(32.7)$ & $18(32.1)$ \\
\hline Duration of RA, mean (SD), years & $8.3(9.0)$ & $9.5(8.9)$ & $11.0(10.0)$ & $10.4(8.6)$ \\
\hline Rheumatoid factor positive, $n(\%)$ & $41(73.2)$ & $34(64.2)$ & $37(71.2)$ & $42(75.0)$ \\
\hline $\begin{array}{l}\text { Anti-CCP autoantibody positive, } \\
n(\%)\end{array}$ & $42(76.4)^{\mathrm{a}}$ & $37(69.8)$ & $35(67.3)$ & $47(83.9)$ \\
\hline TJC, mean $(\mathrm{SD})$ & $23.3(12.7)$ & $24.8(15.3)$ & $25.0(13.2)$ & $24.3(13.9)$ \\
\hline SJC, mean $(S D)$ & $17.4(9.8)$ & $19.3(13.7)$ & $18.2(9.6)$ & $15.4(9.0)$ \\
\hline $\mathrm{CRP}$, mean $(\mathrm{SD}), \mathrm{mg} / \mathrm{L}$ & $16.8(23.6)$ & $17.3(18.8)$ & $20.4(26.6)$ & $21.2(24.2)$ \\
\hline HAQ-DI score, mean (SD) & $1.5(0.7)$ & $1.6(0.7)$ & $1.6(0.6)$ & $1.7(0.7)$ \\
\hline DAS28-CRP, mean (SD) & $5.7(0.8)$ & $5.7(1.0)$ & $5.8(1.0)$ & $5.7(1.0)$ \\
\hline
\end{tabular}

$C C P$ cyclic citrullinated peptide, $C R P$ C-reactive protein, DAS28-CRP 28-joint disease activity score by CRP, DMARD disease-modifying antirheumatic drug, HAQ-DI Health Assessment Questionnaire-Disability Index, $q 2 w$ every 2 weeks, $R A$ rheumatoid arthritis, $S D$ standard deviation, $S J C$ swollen joint count, TJC tender joint count

${ }^{\text {a }} N=55$

\section{Pharmacokinetics}

For each dose group, concentration-time profiles of sarilumab after the first dose (weeks 0-2) and at the sixth dose (weeks 10-12) were comparable between the pen and syringe (Fig. 3), and the area under the plasma concentration-time curves from 0 to the end of the dosing period $\left(\mathrm{AUC}_{0-\tau}\right)$ were similar between the pen and syringe (Table 3).

\section{Safety}

There were no clinically meaningful differences in treatment-emergent AEs (TEAEs), serious AEs (SAEs), or AEs leading to discontinuation among the sarilumab pen and syringe groups (Table 4). Although the incidence of SAEs and
TEAEs leading to discontinuations was numerically higher in the sarilumab $200 \mathrm{mg}$ groups compared with the sarilumab $150 \mathrm{mg}$ groups, the events were distributed across system organ classes with no particular pattern. There were no cases of anaphylaxis, and no hypersensitivity reactions were reported as SAEs. Similar to prior sarilumab studies [2-4], the most common TEAEs were infections and neutropenia. There were no opportunistic infections and only three serious infections (one in the pen $150 \mathrm{mg}$ group and two in the syringe $200 \mathrm{mg}$ group). The overall incidence of injection site reactions was similar in the sarilumab pen and syringe groups, and the most common injection site reaction was injection site erythema (Table 4). Only one patient, from the sarilumab pen $150 \mathrm{mg}$ group, reported injection site pain. 
Table 2 Patient perception and satisfaction with the sarilumab pen

Questions

Answers (patients using

the pen)

Baseline (before first injection)

How afraid are you of needles? ? $^{a}$

58\%: not at all

How confident are you about giving yourself an injection ${ }^{\text {? }}$

55\%: very to extremely

confident

Have you had any experience in performing injections on yourself? ${ }^{b}$

$55 \%$ : yes

For treating your RA?

43\%: yes

For treating a disease other than your RA?

$16 \%$ : yes

Week 12 (after last injection)

How difficult or easy was it to understand the instructions for use?

$88 \%$ : easy to very easy

How difficult or easy was it to remove the cap? ${ }^{c}$

90\%: easy to very easy

How difficult or easy was it to hold the pen in your hand?

$87 \%$ : easy to very easy

How difficult or easy was it to administer the injection without any help? ${ }^{c}$

$88 \%$ : easy to very easy

How did you find the time it took to inject the medication (when the needle was in the skin) $?^{\mathrm{d}}$

Overall, how difficult or easy was it to use this pen ${ }^{c}$

64\%: short to very short

Overall, how satisfied are you with this device?

If you previously used another autoinjector/pen to inject another RA medication, how easy or difficult was it to use the pen in this study in comparison to your previous experience? ${ }^{f}$

After this study, would you choose to self-inject your medication? ${ }^{\text {g }}$

$88 \%$ : easy to very easy

98\%: satisfied to very satisfied

$83 \%$ : easier to much easier

After this study, how confident would you be to give yourself injections using the same type of pen as the one used in this study? ${ }^{\mathrm{a}}$

$72 \%$ : definitely yes

91\%: very to extremely confident

$R A$ rheumatoid arthritis

${ }^{a}$ Not at all/a little/moderately/very/extremely

b Yes/no

c Very difficult/difficult/somewhat difficult/somewhat easy/easy/very easy

d Very long/long/normal/short/very short

e Very dissatisfied/dissatisfied/neither dissatisfied nor satisfied/satisfied/very satisfied

f Much more difficult/more difficult/same/easier/much easier/do not remember

g Definitely not/probably not/I don't know/probably yes/definitely yes

The majority of decreases in absolute neutrophil count (ANC) were between $1.0 \mathrm{Giga} / \mathrm{L}$ and the lower limit of normal (LLN; i.e., grade 1-2 neutropenia; Table 4). Patients with ANC below LLN did not have a higher incidence of infection compared with patients with ANC in the normal range.
Increases in alanine aminotransferase (ALT) were mostly mild. There were no cases of Hy's law, defined as drug-induced liver injury resulting in increased ALT levels $>3 \times$ the upper limit of normal (ULN) and total bilirubin levels $>2 \times$ ULN after excluding other potential causes [16]. 
a

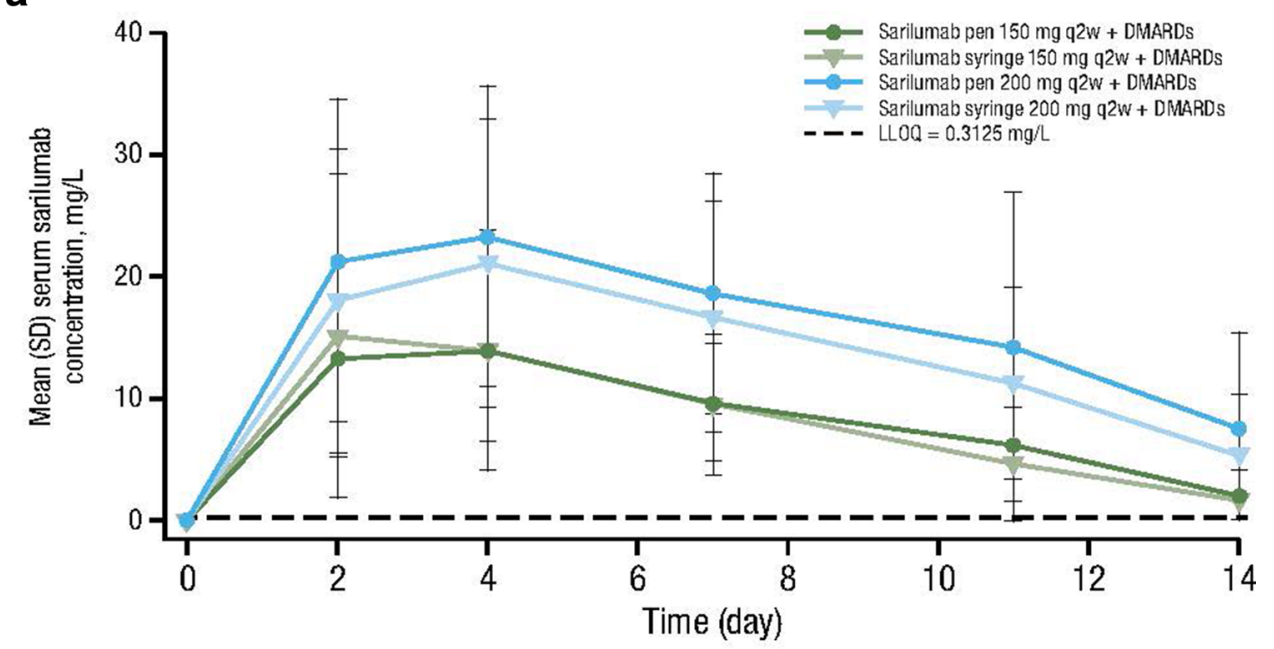

b

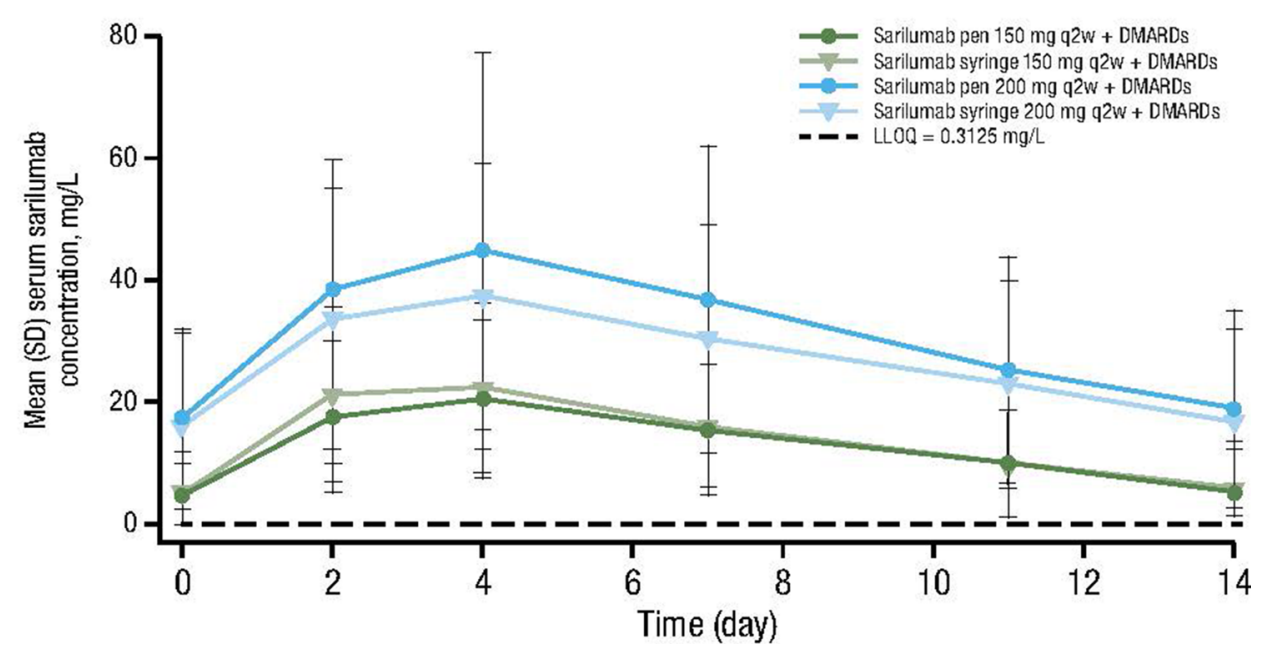

Fig. 3 Mean $( \pm S D)$ serum sarilumab-time profiles. Mean serum sarilumab concentration is shown for the pen and syringe for the 150 and $200 \mathrm{mg} \mathrm{q} 2 \mathrm{w}$ doses at a weeks $0-2$ and b weeks 10-12. DMARD disease-

\section{Efficacy}

The proportion of patients achieving an ACR20 response at week 12 was generally similar across treatment groups and between pen and syringe $(80.4 \%$ and $71.7 \%$ at $150 \mathrm{mg}$, and $69.2 \%$ and $71.4 \%$ at $200 \mathrm{mg}$, respectively). Comparable trends were observed at week 12 with ACR50/70 responses (Fig. 4). The proportion of patients achieving DAS28-CRP $<2.6$ was also generally similar across treatment groups and between modifying antirheumatic drug, $L L O Q$ lower limit of quantification, $q 2 w$ every 2 weeks, $S D$ standard deviation

pen and syringe $(26.8 \%$ and $30.2 \%$ at $150 \mathrm{mg}$, and $30.8 \%$ and $28.6 \%$ at $200 \mathrm{mg}$, respectively).

\section{DISCUSSION}

This phase 3 study demonstrated the ease of use and robustness of the sarilumab pen when used by patients with active moderate-to-severe RA in an unsupervised real-world setting. Patients were provided with either a single-use, disposable, prefilled pen or syringe. The pen serves as a 
Table 3 Pharmacokinetic parameters of the sarilumab pen and syringe

\begin{tabular}{|c|c|c|c|c|}
\hline \multirow[t]{2}{*}{ Parameter $^{\mathrm{a}}$} & \multicolumn{4}{|c|}{ Sarilumab q2w + DMARDs } \\
\hline & Pen $150 \mathrm{mg}$ & Syringe $150 \mathrm{mg}$ & Pen $200 \mathrm{mg}$ & Syringe $200 \mathrm{mg}$ \\
\hline \multicolumn{5}{|l|}{$C_{\max }, \mathrm{mg} / \mathrm{L}$} \\
\hline Week $0-2, n$ & 53 & 51 & 50 & 53 \\
\hline Mean (SD) & $16.5(9.2)$ & $16.7(13.0)$ & $27.6(15.2)$ & $23.7(12.7)$ \\
\hline Week $10-12, n$ & 45 & 44 & 36 & 46 \\
\hline Mean $(S D)$ & $21.7(12.9)$ & $24.2(14.8)$ & $50.4(33.8)$ & $39.4(22.3)$ \\
\hline \multicolumn{5}{|l|}{$t_{\max }$, days } \\
\hline Week $0-2, n$ & 53 & 51 & 50 & 53 \\
\hline Median (range) & $2.9(1.8-11.0)$ & $2.9(0.9-6.9)$ & $3.9(1.9-10.0)$ & $3.7(1.7-10.9)$ \\
\hline Week $10-12, n$ & 45 & 44 & 36 & 46 \\
\hline Median (range) & $3.9(0.0-6.8)$ & $3.1(1.7-7.0)$ & $3.9(1.0-8.0)$ & $3.9(1.0-11.0)$ \\
\hline \multicolumn{5}{|l|}{$\mathrm{AUC}_{0-\tau}, \mathrm{mg}$ day/L } \\
\hline Week $0-2, n$ & 39 & 34 & 34 & 41 \\
\hline Mean (SD) & $131(54.5)$ & $152(76.7)$ & $235(117)$ & $227(94.9)$ \\
\hline Week 10-12, $n$ & 44 & 40 & 36 & 38 \\
\hline Mean (SD) & $205(126)$ & $220(130)$ & $455(294)$ & $405(244)$ \\
\hline
\end{tabular}

$A U C_{0-\tau}$ area under the plasma concentration-time curve from 0 to the end of the dosing period, $C_{\max }$ maximum observed drug concentration, DMARD disease-modifying antirheumatic drug, $q 2 w$ every 2 weeks, $S D$ standard deviation, $t_{\max }$ time to $C_{\max }$

${ }^{a}$ Data were excluded if a patient did not take the study drug or took the wrong dose at day 1 or 71

delivery device and houses the same syringe as the syringe assessed in the comparator group. After 12 weeks of treatment, the primary endpoint was met with no pen-associated PTFs and only one PTC (attributed to user-related error), and $98 \%$ of patients were satisfied to very satisfied with the sarilumab pen. Most patients $(88 \%)$ thought the pen was easy or very easy to use; $98 \%$ thought the injection time was either normal, short, or very short; and $91 \%$ were very to extremely confident about using the same pen for self-injection in the future.

Safety and efficacy appeared to be generally comparable between the pen and syringe and consistent with observations from other clinical trials of sarilumab [2-4]. There were no clinically meaningful differences between the pen and syringe regarding the incidence or types of reported TEAEs, SAEs, or TEAEs leading to discontinuation. Although the incidence of SAEs was numerically higher with sarilumab $200 \mathrm{mg}$ $\mathrm{q} 2 \mathrm{w}$ compared with sarilumab $150 \mathrm{mg} \mathrm{q} 2 \mathrm{w}$, the number of patients with SAEs was small and distributed across system organ classes without any particular pattern. There was also no clinically meaningful difference in the overall incidence of injection site reactions between the pen and syringe. The PK and exposure (maximum observed drug concentration [Cmax] and $\mathrm{AUC}_{0-\tau}$ ) of sarilumab were generally similar after administration with the pen and syringe.

Patients with RA may need to administer biologic therapies via subcutaneous injections and may have difficulty manipulating syringes, as RA often affects the small joints of the hand, impairing dexterity [17]. Results from previous 
Table 4 Summary of AEs, injection site reactions, grade 3/4 neutropenia, and liver function in patients

\section{Sarilumab q2w + DMARDs}

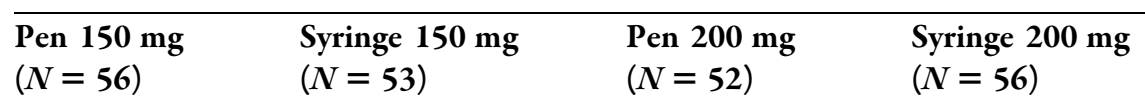

Overview of AEs, $n$ (\%)

$\begin{array}{lllll}\text { TEAEs } & 42(75.0) & 30(56.6) & 34(65.4) & 35(62.5) \\ \text { SAEs } & 1(1.8) & 0 & 3(5.8) & 4(7.1) \\ \begin{array}{l}\text { Discontinuations due to } \\ \text { TEAEs }\end{array} & 3(5.4) & 3(5.7) & 7(13.5) & 3(5.4) \\ \text { Deaths } & 0 & & & 0 \\ \text { TEAEs in }>5 \text { patients, } n \text { (\%) } & & 0 & 0 & 3(5.4) \\ \text { Neutropenia } & 10(17.9) & 9(17.0) & 6(11.5) & 3(5.4) \\ \text { Upper respiratory tract } & 3(5.4) & 2(3.8) & 1(1.9) & 2(3.6) \\ \text { infection } & & & & 2(3.6) \\ \text { Hypertension } & 2(3.6) & 1(1.9) & 3(5.8) & 1(1.8) \\ \text { Nasopharyngitis } & 4(7.1) & 1(1.9) & 1(1.9) & 2(3.6) \\ \text { Thrombocytopenia } & 4(7.1) & 1(1.9) & 2(3.8) & 1(1.8) \\ \text { Urinary tract infection } & 2(3.6) & 2(3.8) & 2(3.8) & 1(1.8) \\ \text { Bronchitis } & 4(7.1) & 1(1.9) & 1(1.9) & 2(3.6) \\ \text { Pharyngitis } & 5(8.9) & 0 & 1(1.9) & 0 \\ \text { Headache } & 2(3.6) & 2(3.8) & & \end{array}$

Most frequent injection site reactions ( $\geq 5 \%$ in at least one treatment group), $n$ (\%)

$\begin{array}{lllll}\text { Any injection site reaction } & 5(8.9) & 3(5.7) & 5(9.6) & 5(8.9) \\ \text { Injection site erythema } & 2(3.6) & 2(3.8) & 4(7.7) & 4(7.1) \\ \text { Injection site pruritus } & 1(1.8) & 2(3.8) & 0 & 4(7.1)\end{array}$

Absolute neutrophil count, $n$ (\%)

Grade 1: $1.5 \mathrm{Giga} / \mathrm{L}$ to LLN $8(14.3)$

$9(17.0) \quad 9(28.8) \quad 9(16.1)$

Grade 2: 1.0 to $<1.5 \mathrm{Giga} / \mathrm{L} \quad 6(10.7)$

Grade 3: 0.5 to $<1.0 \mathrm{Giga} / \mathrm{L} \quad 6(10.7)$

Grade 4: $<0.5 \mathrm{Giga} / \mathrm{L}$

Alanine aminotransferase, $n$ (\%)

\begin{tabular}{lllll}
$>3$ to $5 \times \mathrm{ULN}$ & $1(1.8)$ & 0 & $3(5.8)$ & $1(1.8)$ \\
$>5 \times \mathrm{ULN}$ & 0 & 0 & $1(1.9)$ & 0 \\
\hline
\end{tabular}

$A E$ adverse event, $L L N$ lower limit of normal, $q 2 w$ every 2 weeks, SAE serious AE, TEAE treatment-emergent AE, ULN upper limit of normal 


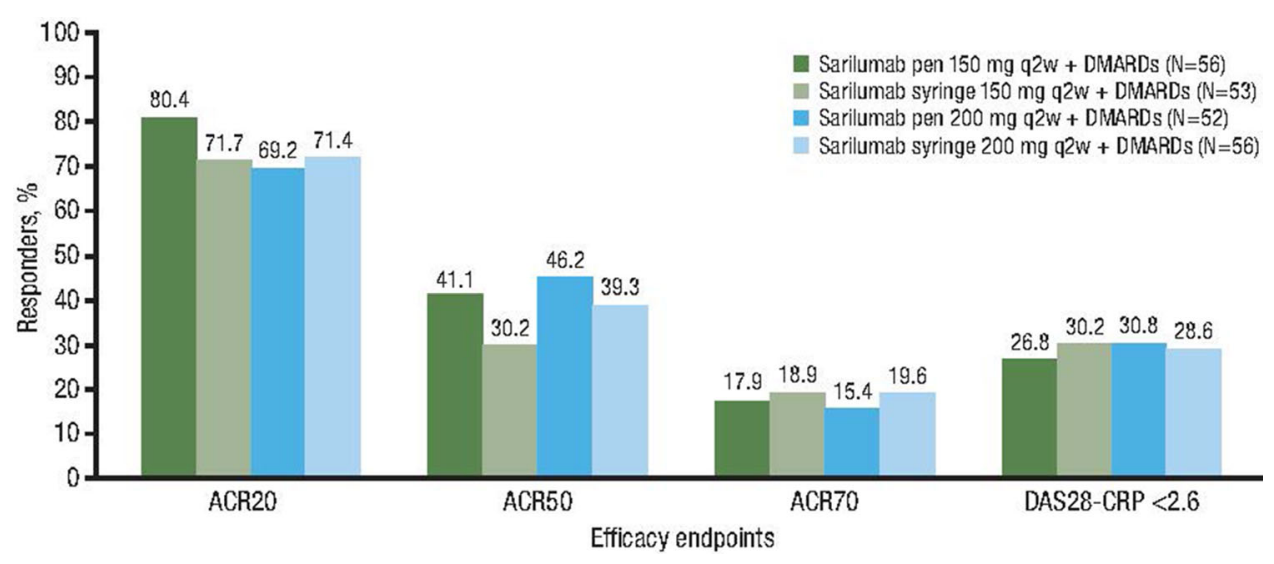

Fig. 4 Proportions of patients achieving an ACR20/50/ 70 response and DAS28-CRP $<2.6$ at week 12. $A C R$ American College of Rheumatology, DAS28-CRP 28-joint

studies have shown that patients with RA favor treatment devices that are easy to use, convenient, less painful, and take less time to use, and patients have demonstrated a preference for autoinjector devices over more conventional methods of treatment administration $[13,18,19]$, such as syringes. Pens have been well accepted for the treatment of other chronic health conditions, including diabetes mellitus, migraine headaches, and growth hormone deficiency, and subcutaneous administration of a tumor necrosis factor (TNF) inhibitor via pen has also been accepted for the treatment of RA [13]. As RA requires lifelong treatment, the use of a pen that is ergonomically designed to take into account the manual dexterity issues relevant to this patient population could potentially enhance compliance. Beyond its convenience, the pen also offers safety protection features that prevent needlestick injury before and after use.

\section{CONCLUSIONS}

In this study, patients with RA successfully completed self-injections with a single-use, disposable, prefilled, buttonless, ergonomic sarilumab pen. Patients reported satisfaction with the sarilumab pen and found the pen easy to use. In addition, patients were very confident regarding the use of such a pen for self-injection disease activity score by $\mathrm{C}$-reactive protein, DMARD disease-modifying antirheumatic drug, $q 2 w$ every 2 weeks

in the future. Safety and efficacy appeared to be generally similar between the pen and syringe groups and consistent with observations from other clinical trials of sarilumab. In conclusion, this study demonstrated the ease of use and robustness of sarilumab 150 and $200 \mathrm{mg}$ q2w administered via pen when used by patients with RA in an unsupervised real-world setting.

\section{ACKNOWLEDGEMENTS}

Funding. This study was sponsored by Sanofi Genzyme (Bridgewater, NJ, USA) and Regeneron Pharmaceuticals, Inc. (Tarrytown, NY, USA). Article processing charges were funded by Sanofi Genzyme (Bridgewater, NJ, USA) and Regeneron Pharmaceuticals, Inc. (Tarrytown, NY, USA). All authors had full access to all of the data in this study and take complete responsibility for the integrity of the data and accuracy of the data analysis.

Authorship. All named authors meet the International Committee of Medical Journal Editors (ICMJE) criteria for authorship for this manuscript, take responsibility for the integrity of the work as a whole, and have given final approval to the version to be published. 
Medical Writing, Editorial, and Other Assistance. The authors would like to thank Maria Rell-Bakalarska, MD, Rheumatology and Osteoporosis Outpatient Clinic, for reviewing the data, and Anne Paccaly, PharmD, PhD, Regeneron Pharmaceuticals, Inc., for critically reviewing the data and manuscript. Editorial assistance was provided under the direction of the authors by Allison Rosenberg, PhD, and Jennifer Rossi, MA, ELS, MedThink SciCom, and funded by Sanofi Genzyme and Regeneron Pharmaceuticals, Inc.

Disclosures. Alan Kivitz has received consulting fees from AbbVie, Boehringer Ingelheim, Novartis, Sanofi, Pfizer, Roche, and UCB. Lydie Baret-Cormel is an employee of Sanofi and may hold stock and/or stock options in the company. Hubert van Hoogstraten is an employee of Sanofi Genzyme and may hold stock and/or stock options in the company. Sheldon Wang is an employee of Sanofi Genzyme and may hold stock and/or stock options in the company. Christine $\mathrm{Xu}$ is an employee of Sanofi Genzyme and may hold stock and/or stock options in the company. Janie Parrino is an employee of Regeneron Pharmaceuticals, Inc., and may hold stock and/or stock options in the company. Marina Stanislav declares that she has no conflict of interest.

Compliance with Ethics Guidelines. All procedures followed were in accordance with the ethical standards of the responsible committee on human experimentation (institutional and national) and with the Helsinki Declaration of 1964, as revised in 2013. Informed consent was obtained from all patients before they were included in the study.

Open Access. This article is distributed under the terms of the Creative Commons Attribution-NonCommercial 4.0 International License (http://creativecommons.org/licenses/ by-nc/4.0/), which permits any noncommercial use, distribution, and reproduction in any medium, provided you give appropriate credit to the original author(s) and the source, provide a link to the Creative Commons license, and indicate if changes were made.

\section{REFERENCES}

1. Scott DL, Wolfe F, Huizinga TW. Rheumatoid arthritis. Lancet. 2010;376:1094-108.

2. Burmester GR, Lin Y, Patel R, et al. Efficacy and safety of sarilumab monotherapy versus adalimumab monotherapy for the treatment of patients with active rheumatoid arthritis (MONARCH): a randomised, double-blind, parallel-group phase III trial. Ann Rheum Dis. 2017;76:840-7.

3. Fleischmann R, van Adelsberg J, Lin Y, et al. Sarilumab and nonbiologic disease-modifying antirheumatic drugs in patients with active rheumatoid arthritis and inadequate response or intolerance to tumor necrosis factor inhibitors. Arthritis Rheumatol. 2017;69:277-90.

4. Genovese MC, Fleischmann R, Kivitz AJ, et al. Sarilumab plus methotrexate in patients with active rheumatoid arthritis and inadequate response to methotrexate: results of a phase III study. Arthritis Rheumatol. 2015;67:1424-37.

5. Dayer JM, Choy E. Therapeutic targets in rheumatoid arthritis: the interleukin-6 receptor. Rheumatology (Oxf). 2010;49:15-24.

6. Sugiyama E, Kuroda A, Hori F, et al. Serum interleukin-6 level is a sensitive parameter of disease activity in rheumatoid arthritis. J Clin Rheumatol. 1995;1:93-8.

7. Maini RN, Taylor PC, Szechinski J, et al. Doubleblind randomized controlled clinical trial of the interleukin-6 receptor antagonist, tocilizumab, in European patients with rheumatoid arthritis who had an incomplete response to methotrexate. Arthritis Rheum. 2006;54:2817-29.

8. Smolen JS, Beaulieu A, Rubbert-Roth A, et al. Effect of interleukin-6 receptor inhibition with tocilizumab in patients with rheumatoid arthritis (OPTION study): a double-blind, placebo-controlled, randomised trial. Lancet. 2008;371:987-97.

9. Jones $\mathrm{G}$, Sebba A, Gu J, et al. Comparison of tocilizumab monotherapy versus methotrexate monotherapy in patients with moderate to severe rheumatoid arthritis: the AMBITION study. Ann Rheum Dis. 2010;69:88-96.

10. Maini RN, Breedveld FC, Kalden JR, et al. Therapeutic efficacy of multiple intravenous infusions of anti-tumor necrosis factor $\alpha$ monoclonal antibody combined with low-dose weekly methotrexate in rheumatoid arthritis. Arthritis Rheum. 1998;41:1552-63. 
11. Bartelds GM, Wijbrandts CA, Nurmohamed MT, et al. Clinical response to adalimumab: relationship to anti-adalimumab antibodies and serum adalimumab concentrations in rheumatoid arthritis. Ann Rheum Dis. 2007;66:921-6.

12. Schaeverbeke $\mathrm{T}$, Truchetet ME, Kostine M, Barnetche $\mathrm{T}$, Bannwarth $\mathrm{B}$, Richez $\mathrm{C}$. Immunogenicity of biologic agents in rheumatoid arthritis patients: lessons for clinical practice. Rheumatology (Oxf). 2016;55:210-20.

13. Kivitz A, Cohen S, Dowd JE, et al. Clinical assessment of pain, tolerability, and preference of an autoinjection pen versus a prefilled syringe for patient self-administration of the fully human, monoclonal antibody adalimumab: the TOUCH trial. Clin Ther. 2006;28:1619-29.

14. Aletaha D, Neogi T, Silman AJ, et al. 2010 Rheumatoid arthritis classification criteria: an American College of Rheumatology/European League Against Rheumatism collaborative initiative. Arthritis Rheum. 2010;62:2569-81.

15. Hochberg MC, Chang RW, Dwosh I, Lindsey S, Pincus T, Wolfe F. The American College of Rheumatology 1991 revised criteria for the classification of global functional status in rheumatoid arthritis. Arthritis Rheum. 1992;35:498-502.

16. Robles-Diaz M, Lucena MI, Kaplowitz N, et al. Use of Hy's law and a new composite algorithm to predict acute liver failure in patients with drug-induced liver injury. Gastroenterology. 2014;147:109-18.

17. Schiff M, Koo J, Jin E, et al. Usability and acceptability of the abatacept pre-filled autoinjector for the subcutaneous treatment of rheumatoid arthritis. Adv Ther. 2016;33:199-213.

18. Demary W, Schwenke H, Rockwitz K, et al. Subcutaneously administered methotrexate for rheumatoid arthritis, by prefilled syringes versus prefilled pens: patient preference and comparison of the selfinjection experience. Patient Prefer Adherence. 2014;8:1061-71.

19. Thakur K, Biberger A, Handrich A, Rezk MF. Patient perceptions and preferences of two etanercept autoinjectors for rheumatoid arthritis: findings from a patient survey in Europe. Rheumatol Ther. 2016;3:245-56. 